\title{
An Optimized Method Based on Digitalized Lissajous Curve to Determine Lifetime of Luminescent Materials on Optical Fiber Sensors
}

\author{
Adrián Ridruejo, Nerea De Acha, César Elosúa, Ignacio R. Matías, and Francisco J. Arregui \\ Department of Electrical and Electronic Engineering, Public University of Navarra, 31006 Pamplona, Spain \\ Correspondence should be addressed to César Elosúa; cesar.elosua@unavarra.es
}

Received 3 January 2016; Revised 29 April 2016; Accepted 10 May 2016

Academic Editor: Hai Xiao

Copyright (C) 2016 Adrián Ridruejo et al. This is an open access article distributed under the Creative Commons Attribution License, which permits unrestricted use, distribution, and reproduction in any medium, provided the original work is properly cited.

\begin{abstract}
A method is proposed to determine lifetime of luminescent emissions based on the phase shift measurement employing the digitalized Lissajous representation: this diagram has been typically used with analogical algorithms, whereas the proposed method is performed in digital domain, showing an improved accuracy and repeatability. The procedure is studied and tested with two different oxygen sensors that show different sensitivities and signal levels in order to confirm the no influence of the signals intensity on the calibration process. The computational cost of the proposed method is low, which makes it possible to monitor in real time luminescence sensors based on reversible quenching with a potential low cost system based on a digital signal processor (DSP).
\end{abstract}

\section{Introduction}

Technological research assigns many resources to the development of sensors for a specific purpose, which is providing more independency to the products and systems that improve human quality of live. Nowadays, smart cities require the information of sensors of different types to enhance the performance of the urban services, reducing cost and pollution as well as using natural resources more efficiently [1]. To be able to react against changes in the environment, it is needed to monitor every parameter involved: that is the reason why sensors take on a special relevancy in these systems. Sensors are devices able to sense a wide range of different magnitudes, so the information provided by them has to be registered and processed by the systems to offer an answer as better as possible.

Therefore, it is important to have sensors able to measure distinct physical or chemical magnitudes. Furthermore, each type of sensor needs a processing unit that can register and monitor the transduction between the measurement and the parameter recorded by the sensor. This step can be as critical as the sensor construction itself and determines drastically the whole performance of the system. In this context, optical fiber sensors show a relevant potential due to the advantages of using optical fiber against electric cables in several scenarios $[2,3]$. However, the implementation in real applications is not as extended as it could be, because there are effects which modify the light behavior along the time: one of the most challenging ones is produced by undesired artifacts in the intensity of the sensor signal when the transduction is based on this parameter.

Luminescent sensors register the emission of a specific material when it is illuminated with a light source at a certain wavelength. The transduction takes place when the target magnitude modifies the properties of the sensing material emission, for example, its intensity [4]. One way to detect this variation consists of coupling it into an optical fiber and then registering it. In most cases, the chemical dye suffers a quenching that alters the lifetime of the fluorescent emission, which is the mean time that an electron takes to recover its quiescent state (at the lowest conducting band) from an excited one [5]. Materials such as metallic porphyrins show this behavior in the presence of some gases: one of the most relevant applications where they are used is oxygen detection and concentration measurement [6]. For this specific case, lifetime is reduced as the gas concentration 
increases: this effect is directly measurable by the magnitude of the luminescent emission, which is used in intensity based sensors [7-9]. However, there are many artifacts that can modify the intensity level, altering the final measurement as well as photobleaching [10]. This dependence on the signal level limits the use of intensity based sensors in real scenarios.

There are approaches that analyze other parameters in order to overcome this problem: lifetime is an interesting alternative because it is a temporal parameter and therefore it is not affected by signal level fluctuations [11, 12]. This parameter can be obtained by measuring other ones that depend directly on it: in the case the exciting signal is modulated, the luminescent emitted one is also modulated; therefore, the phase shift between them is defined by the lifetime value $[13,14]$. Moreover, in case the modulation is sinusoidal [15], the relation between the phase shift and the lifetime can be described as shown in

$$
\tan \varphi=2 \pi f \tau
$$

where $\varphi$ is the phase shift, $f$ is the frequency of the exciting signal, and $\tau$ is the luminescence lifetime. As it can be inferred, when using this relation, intensity fluctuations have no influence, so that the system becomes more robust. In most cases, the luminescent signal shows a lower signal-tonoise ratio (SNR) compared to the excitation one, so that it has to be properly conditioned to be able to measure the phase shift between the signals. Typically, the emitted signal is converted to the electrical domain to be regenerated by a lock-in amplifier, and then, it is digitalized to be processed in the discrete time domain [16]. In this manner, the lockin amplifier is required to measure the phase shift, so that this procedure is not able to handle directly with the low SNR electric signal obtained from the sensor. Alternatives based on nonstandard fibers or complex methods to excite the sensor have been developed trying to avoid the use of this kind of instrumentation [17]; however, the high cost of these devices compromises the potentiality of the final system.

In this paper, a fluorescence lifetime system is proposed based on phase shift measurement, so it is independent on the light intensity. A specific algorithm has been developed to calculate the phase shift: it works with the Lissajous curve in the digital domain and it handles the low level signal emitted by the sensing material; it improves the results obtained by traditional methods such as Fast Fourier Transform (FFT) or zero crossing. Moreover, the calculations are done entirely in the digital domain, highlighting the originality of the work, because these techniques were traditionally employed in the analog domain. Therefore, the whole signal processing (even the modulation of the interrogating signal) can be performed by a single embedded system such as DSP without any signal conditioning in the analogic domain. The proposed method is focused on obtaining lifetime measurements independent of artifacts under conditions where the signal level is low and noisy. The algorithm has been optimized in terms of standard deviation (measured in degrees) following five different implementations. The procedure has been applied to two different optical fiber sensors with different sensitivities to measure distinct oxygen concentrations: although one of them showed a significantly lower signal, they both were correctly calibrated in the $0-20 \%$ oxygen range, which verifies the robustness of the proposed method.

\section{Materials and Methods}

2.1. Sensing Material. In this work, two sensors with a different behavior have been implemented and studied, providing two scenarios well differentiated. They have been prepared with the same sensing material, which was platinum tetrakis pentafluorophenyl porphine (Pt-TFPP). When this product is illuminated with a light source centered on $395 \mathrm{~nm}$, it shows a luminescent emission located at $650 \mathrm{~nm}$. Furthermore, due to its chemical structure, the compound is not soluble in water. The lifetime of this emission depends on the environmental oxygen concentration by a quenching effect, which is reversible [18]. This porphine has been used to develop oxygen sensors on different substrates and transduction principles, based on either intensity modulation $[19,20]$ or lifetime measurement [21]. The behavior of the sensors developed with this material has been described with the Stern-Vollmer equation [22]. Moreover, it shows an optimal thermal and chemical stability [23], so that it was chosen to prepare the sensors to test the performance of the algorithm under study.

2.2. Sensors Construction Process. Two oxygen sensors were implemented with the same sensing material, but using distinct supporting matrices to attach it onto the optical fiber. All the chemical compounds employed were bought from Sigma Aldrich but the Pt-TFPP from Frontier Scientific: all of them were used without any purification. Before the deposition of the sensing material, the fibers were cleaned with a $1 \mathrm{M}$ potassium hydroxide $(\mathrm{KOH})$ aqueous solution.

The supporting matrix used to implement the first probe, named Sensor A, was a plastic one; specifically, polyvinyl chloride (PVC) was used as polymer [24]. For its fabrication, $6 \mathrm{mg}$ of Pt-TFPP, $160 \mathrm{mg}$ of PVC, and $320 \mu \mathrm{L}$ of tributylphosphate (TBP) were dissolved in $4 \mathrm{~mL}$ of tetrahydrofuran. The mixture was sonicated for 30 minutes to get it as uniform as possible [25]. The deposition of the sensing layer was made by the dip-coating method: the fiber was dipped and removed from the cocktail at a constant velocity of $11 \mathrm{~mm} / \mathrm{s}$.

The second sensor was prepared following Layer-byLayer (LbL) method. Briefly, this procedure is based on the assembly of polymer chains with an electrical charge by electrostatic forces [26]. In this manner, the substrate (the optical fiber pigtail) is dipped alternatively into a polycationic and a polyanionic water solution so that once the polymer chains get assembled, they form a bilayer. The most relevant construction parameters of this method are the concentrations of the polymer solutions, their respective $\mathrm{pH}$, and the number of bilayers assembled onto the substrate [27]. The sensor was prepared employing Polyallylamine Hydrochloride (PAH) as polycation in a $10 \mathrm{mM}$ aqueous solution in which $\mathrm{pH}$ was set at 10. The nonmiscibility in water of the sensing material was overcome by preparing negatively charged micelles: to achieve it, $0.4 \mathrm{mg}$ of Pt-TFPP was firstly dissolved in $1 \mathrm{~mL}$ of acetone and thereafter, in $9 \mathrm{~mL}$ of a $10 \mathrm{mM}$ Sodium Dodecyl 


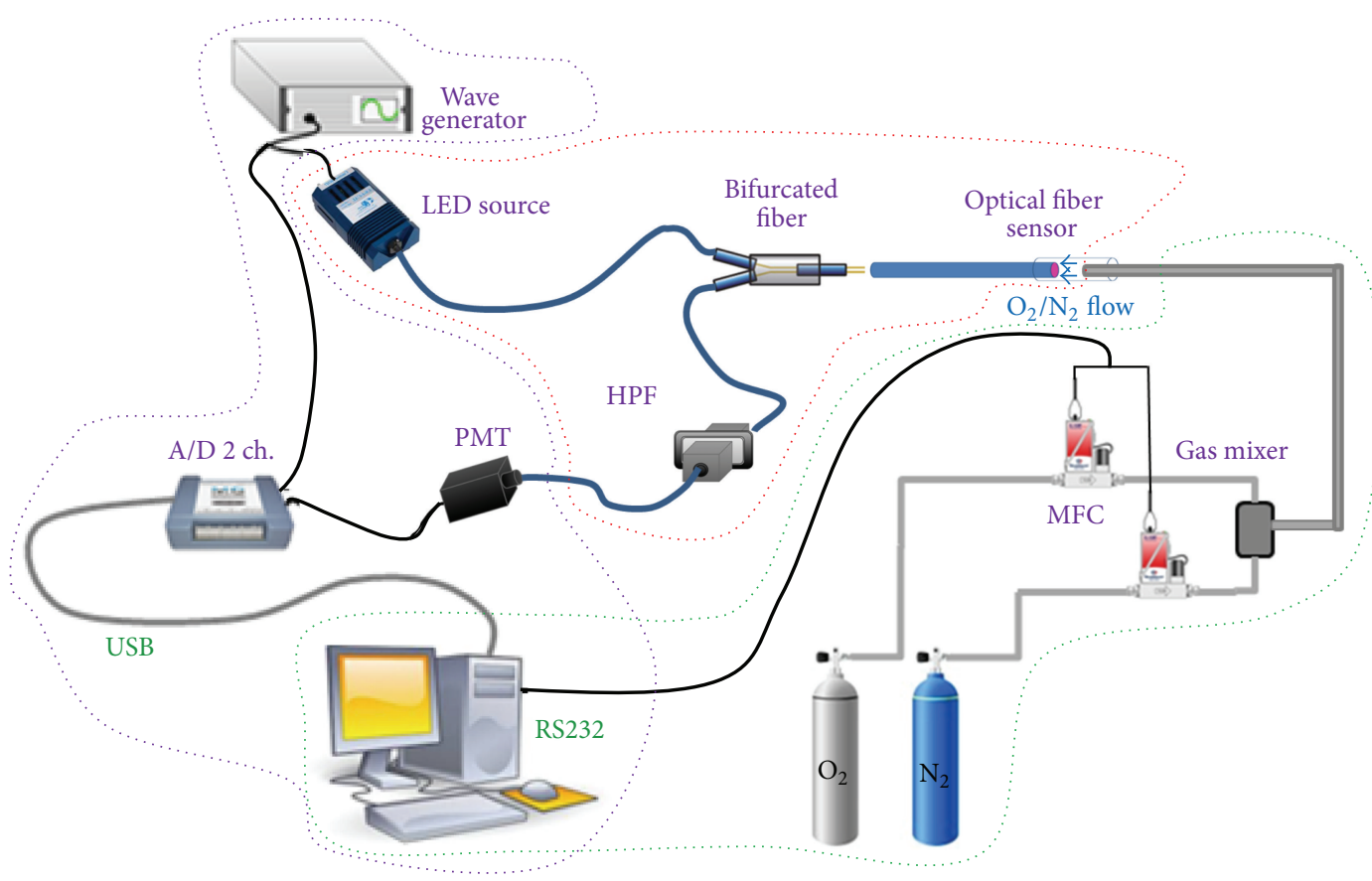

Figure 1: Experimental set-up used for the experiments. The three main blocks are surrounded by colored dotted lines: (red) optical fiber sensor configuration; (green) oxygen flow system; and (purple) electronic devices used for signal modulation and conditioning. The whole system is controlled by a personal computer.

Sulfate (SDS, which acts as anionic surfactant) solution. Due to the negative charged micelles present in this last solution, it was considered as the anionic one. Each bilayer was built following these steps: optical fiber was firstly dipped for 2 minutes in the PAH solution and then rinsed for 1 minute in ultrapure water; thereafter, it was immersed for 4 minutes in the SDS and Pt-TFPP emulsion and washed again for 1 minute in ultrapure water [28]. The immersion into the different solutions was controlled by a programmable robotic arm obtained from Nadetech Innovations S.L. This process was repeated until 40 bilayers were built. The probe prepared in this way was called Sensor B.

\section{Experimental Set-Up}

The experimental set-up can be divided into three main blocks: the first one is related to the optical fiber sensor; the second one includes the electronic devices required to modulate the excitation signal and conditioning the emitted one; the third one is formed by the valves and the system that sets the different oxygen concentrations. A scheme of the whole set-up is shown in Figure 1.

The fiber used to prepare the sensors is Plastic Cladding Silica (PCS), whose core has a diameter of $1000 \mu \mathrm{m}$ (UMT1000), and it was acquired from Thorlabs. The sensor was connected to a $600 \mu \mathrm{m}$ core bifurcated optical fiber (QBIF600-UV-VIS) purchased from Ocean Optics Inc. to obtain the best signal coupling [28]. Following a reflection configuration, the exciting signal from the LED source travels to the sensor head through one of the branches of the bifurcated fiber; meanwhile, the reflected signal from the sensor is guided back through the other branch towards the optical receiver, as well as a nonnegligible contribution from the LED source. In order to attenuate the remaining excitation signal from the sensor response, the second branch of the bifurcated fiber was connected to a high pass filter (LVF-H, also bought at Ocean Optics) whose cutoff wavelength was set at $600 \mathrm{~nm}$.

The excitation signal was modulated at a frequency of $500 \mathrm{~Hz}$ with a wave generator Tektronix CFG280 by a $1 \mathrm{~V}$ peak to peak sinusoidal signal (to modulate the intensity of the LED emission) with a $+3 \mathrm{~V}_{\mathrm{DC}}$ offset (to set the LED conducting). This modulating frequency was chosen because for a certain oxygen concentration (room conditions, $21 \%$ ), it was checked that this value produced the highest phase shift. The optical signal from the HPF is taken into a photomultiplier from Hamamatsu (module H6780-20) to increase its amplitude. The amplified signal was converted to the digital domain and acquired by a USB-202 A/D 2 channel converter from measurement computing. Its sampling frequency was $50 \mathrm{KHz}$. The data were analyzed by Labview ${ }^{\circledR}$ virtual instruments.

The gas flow used to interrogate the sensors was a mixture of gaseous oxygen and nitrogen. The flow rate was similar for all the experiments, and it was set at $250 \mathrm{~mL} / \mathrm{min}$. The composition of the flow (expressed in oxygen \%) was controlled with $\mu$-mass flow valves from Bronkhorst Inc., so that the sum of the flow rate of both gases was always $250 \mathrm{~mL} / \mathrm{min}$ : altering the rate of each gas, different oxygen concentrations could be set. The accuracy of the devices is $0.1 \%$, so that the precision of the different oxygen concentrations was $0.1 \%$. Both gas flows were driven into a mixing chamber and from this point, towards the corresponding optical fiber sensor. Electronic 


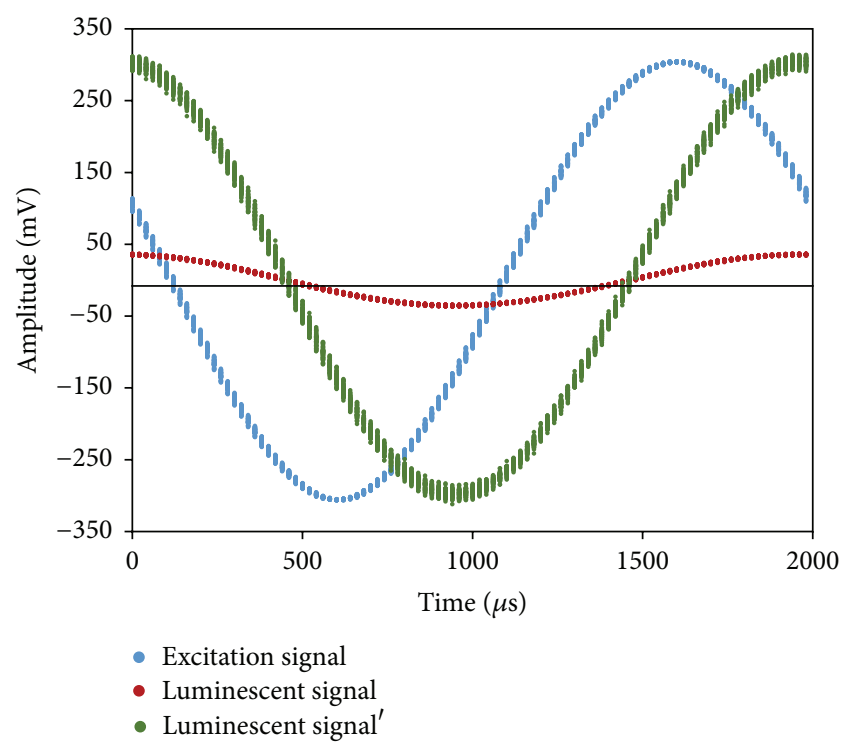

(a)

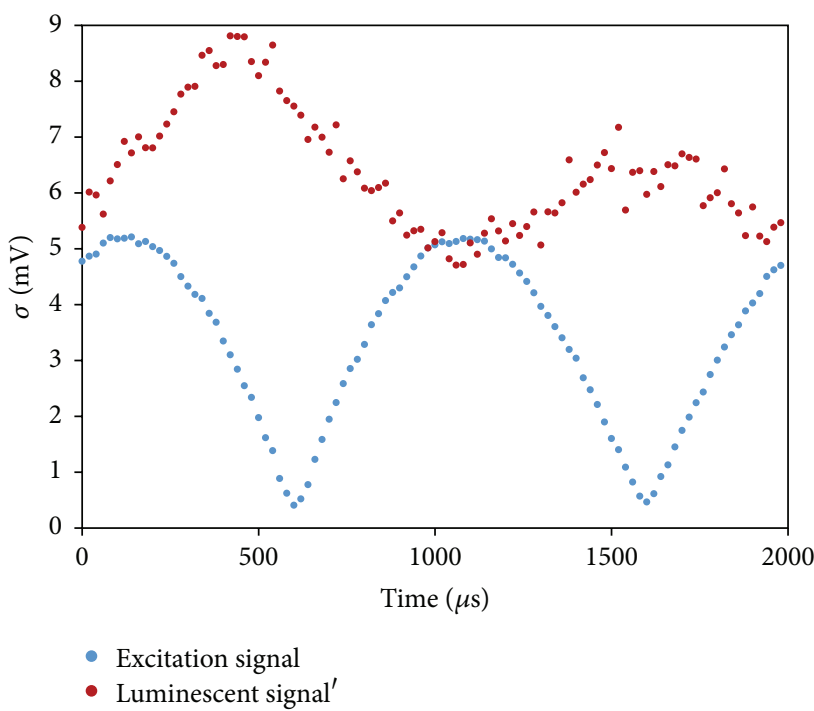

(b)

Figure 2: (a) Excitation signal (blue) and luminescent signal before (red) and after weighing it (green) so both of them show a similar RMS value. (b) Standard deviation expressed in $\mathrm{mV}$ for each sample of the signals under study. The values corresponding to the maximum, minimum, and zero crossing for each signal are pointed for each signal.

sensors could be used to measure the real concentration, but the ratio set by the mass flow controllers was found enough to estimate the actual concentration.

\section{Phase Shift Measurement Methods}

The objective of the procedure is to determine the phase shift between the exciting and the luminescent signals and then use this parameter to estimate the lifetime emission. Both the excitation and luminescent signals were analyzed by averaging 30 cycles in each case. The number of averaged cycles was optimized by considering a different number of cycles (from 1 up to 55) evaluating the resulting standard deviation for each case. The luminescent signal was chosen to estimate the optimal number of cycles to be sampled because it is weaker than the exciting one. It was found that the standard deviation reached a minimum for 30 sampled cycles, and its value was slightly increased of a higher number of averaged samples (as it can be observed in Figure A1 in Supplementary Material available online at http://dx.doi.org/10.1155/2016/6019439); therefore, in order to reduce the preprocessing time, the number of averaged cycles was set at 30 .

The description of the proposed method is based on the signals registered from Sensor A at room conditions (21\% oxygen concentration) and they are displayed in Figure 2(a) (excitation signal in blue, registered luminescent signal in red, and weighted luminescent signal in green): if excitation and luminescent signals are compared, it is evident that the second one shows a much lower amplitude. Moreover, the standard deviation $\sigma$ of both signals was analyzed in order to find the points with the lowest value because they would be optimal to perform the algorithm. The standard deviation from the different samples of both signals is plotted in Figure 2(b). It can be checked that the standard deviation is lower when each signal reaches its maximum or minimum amplitude compared to the zero crossing points: this behavior can be caused by the trigger performance, a small variation of the excited signal frequency or the sample frequency or due to a DC variation. Under these circumstances, estimating the phase shift based on the zero crossing points would yield a high error rate, as it will be checked later.

The proposed method to measure the phase shift between the excitation signal and the luminescent one is based on the Lissajous curve, which is firstly applied for this type of sensors [29]. The Lissajous diagram is the representation of the parametric equation system which describes the superposition of two simple harmonic signals as follows:

$$
\begin{aligned}
& X(t)=A_{1} \cdot \sin \left(2 \pi f_{1} \cdot t+\varphi_{1}\right), \\
& Y(t)=A_{2} \cdot \sin \left(2 \pi f_{2} \cdot t+\varphi_{2}\right) .
\end{aligned}
$$

In our scenario, $X(t)$ is the excitation signal and $Y(t)$ is the luminescent one; $A_{1}$ and $A_{2}$ are the amplitudes of the excitation and emitted signals, respectively; the signals show a similar frequency $f_{1}=f_{2}=f$ but a phase shift between them which is $\varphi=\varphi_{2}-\varphi_{1}$.

The representation $X / Y$ of the parametric equations shows an ellipse where $\varphi$ can be obtained solving (2) when $X=0$ and $Y=0$, respectively:

$$
\begin{aligned}
& Y=0 \longrightarrow X_{0}= \pm A_{1} \cdot \sin (\varphi), \\
& X=0 \longrightarrow Y_{0}= \pm A_{2} \cdot \sin (\varphi), \\
& \varphi=\arcsin \left(\frac{Z_{0}}{A}\right),
\end{aligned}
$$


where $Z_{0}$ can show the values $X_{0}$ or $Y_{0}$ and $A$ does so with $A_{1}$ and $A_{2}$. The phase shift has two possible solutions: to know which is the correct one, it is only needed to check which quadrants are crossed by the major axis of the ellipse [30].

The noise present in the signals is supposed to be Gaussian: therefore, it was decided to evaluate the root mean square (RMS) of both digitalized signals, using the 30 sampled cycles to calculate it (this number period is enough to minimize the noise effect over the RMS value). Once these parameters were obtained, the luminescent signal was weighed in order to adjust its RMS value to the one of the excitation signal. The resulting signal is displayed in Figure 2(a). According to these data, there is an important difference between both signals: the maximum/minimum deviation in the excitation signal is 10 times lower than that at the zero crossing; in the case of the luminescent signal the deviation is of the same order in the three points under study. As it was excepted, the first signal is less noisy, and the maximum minimum points show the lowest deviation: therefore, working with them would yield results with high precision.

Taking into account that the excitation signal shows a lower noise level at the maximum and minimum points, the parametric equations can be rewritten considering $X(t)$ like a nonnoise signal for the equations; in this manner, the resulting equations are

$$
\begin{aligned}
& X(t)=A_{1} \cdot \sin (2 \pi f \cdot t), \\
& Y(t)=A_{2} \cdot \sin (2 \pi f \cdot t+\varphi)+N_{0}(t) .
\end{aligned}
$$

At this point, the signals are sinusoidal and with the same RMS value, so that it can be assumed that both of them have the same maximum amplitude neglecting the Gaussian noise effect from the sensing signal. Therefore, if the phase shift is obtained from the ratio between the maximum and minimum of both signals, the precision of the phase shift estimation would be improved. The digitalized Lissajous curve obtained from the signals with the same RMS value is displayed in Figure 3: the different sampled cycles are superposed to remark the variability of the signal; it can be observed that the lowest values of the deviation are obtained for the distances $X_{\max }$ and $X_{\max }^{\prime}$. All the cycles were averaged and the resulting one is also plotted in the graph: this last representation was the one used to determine the values of the distances $X_{\max }, X_{\max }^{\prime}, Y_{\max }$, or $Y_{\max }^{\prime}, X_{0}, X_{0}^{\prime}, Y_{0}$, or $Y_{0}^{\prime}$ in the digital domain (also indicated in Figure 3 ).

Equation (5) shows how to calculate the phase shift between the signals once the ellipse has been built: there are different possibilities to get the phase shift value depending on the parameters $Z_{0}\left(X_{0}, X_{0}^{\prime}, Y_{0}\right.$, or $\left.Y_{0}^{\prime}\right)$ and $A\left(X_{\max }, X_{\max }^{\prime}\right.$, $Y_{\max }$, or $\left.Y_{\max }^{\prime}\right)$. Figure 3 shows that the signal variation is higher for $Y_{\max }$ or $Y_{\max }^{\prime}$ compared to the values of $X_{\max }$ or $X_{\max }^{\prime}$ : in this background, if it was assumed that the amplitude is similar for both signals because they show the same RMS value (as well as a similar frequency), then $X_{\max }, X_{\max }^{\prime}$ could be used to estimate the phase shift instead of $Y_{\max }$ or $Y_{\max }^{\prime}$, which would yield a lower error rate in the final result.

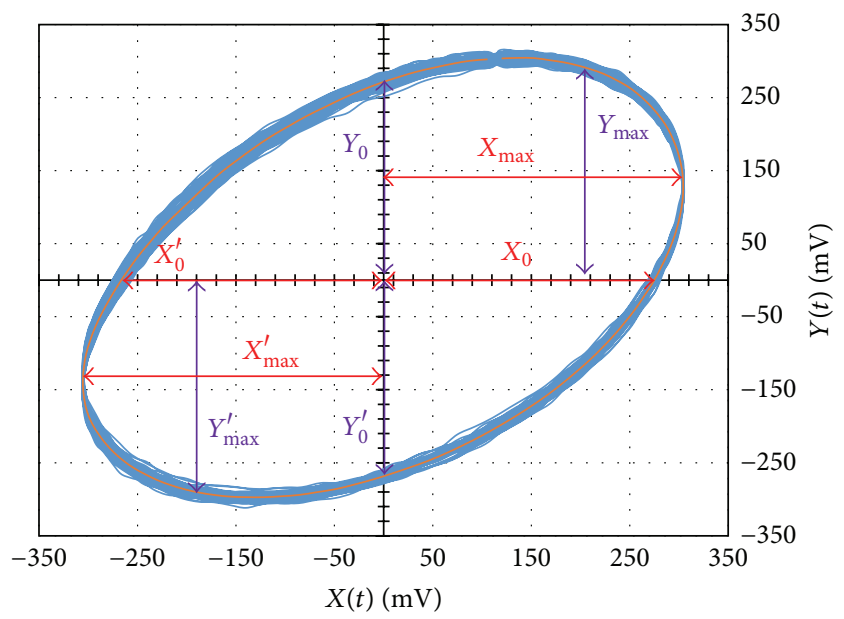

FIGURE 3: Lissajous parametric representation for excitation and weighed luminescent signal. The distances required to calculate the phase shift are indicated.

Taking this hypothesis into account, the following approaches (summarized in Table 1) were proposed.

Method $1 X$. The set of values of $X_{0}$ and $X_{0}^{\prime}$ of $X_{\max }$ and $X_{\max }^{\prime}$ are used to estimate $Z_{0}$ and $A$, respectively. This approach only takes into account the significant points of excitation signal, specifically the ones with the lowest standard deviation $\left(X_{\max }\right.$ and $\left.X_{\max }^{\prime}\right)$ and the ones with the highest value $\left(X_{0}\right.$ and $\left.X_{0}^{\prime}\right)$.

Method $2 X$. The phase shift is calculated twice, firstly using $X_{0}$ and $X_{\max }$ and the other one using $X_{0}^{\prime}$ and $X_{\max }^{\prime}$. Finally, the average value between them determines $\varphi$.

Method $1 Y$. This method is similar to the first one proposed, but the different values are obtained from the luminescent signal, which shows worse standard deviation values.

Method $2 Y^{\prime}$. The approach combines parameters from both the excitation and the luminescent signals, assuming that they both have a similar RMS value after weighting the last one. In this manner, $\varphi$ is calculated twice, firstly using $Y_{0}$ and $X_{\max }$ and secondly employing $Y_{0}^{\prime}$ and $X_{\max }^{\prime}$. The angles obtained are averaged to get the phase shift.

Method $1 X^{\prime}-1 Y^{\prime}$. Two phase shifts are estimated. The first one is obtained applying method $1 X$, whereas for the second one, $Z_{0}^{\prime}$ is the mean of $Y_{0}$ and $Y_{0}^{\prime} ; A$ is calculated as averaged value between $X_{\max }$ and $X_{\max }^{\prime}$ following the hypothesis that the signals show the same RMS value, are sinusoidal, and, therefore, have an equal amplitude. The final value is the average of these two phase shifts.

The results for the distinct methods just described for Sensor $\mathrm{A}$ at room conditions are around $72^{\circ}$. The phase shift has an offset component due to time delay produced by the high pass filter, the photo multiplier, and the electronic instrumentation: this parameter has to be measured and compensated from the measurements in order to get 
TABLE 1: Mathematical expressions to determine the phase shift by the proposed methods based on the Lissajous curve.

\begin{tabular}{lr}
\hline Method $1 X$ & Phase shift \\
\hline Method $2 X$ & $\varphi=\arcsin \left(\frac{X_{0}+X_{0}^{\prime}}{X_{\max }+X_{\max }^{\prime}}\right)$ \\
\hline Method $1 Y$ & $\varphi=\arcsin \left(\frac{Y_{0}+Y_{0}^{\prime}}{Y_{\max }+Y_{\max }^{\prime}}\right)$ \\
\hline Method $2 Y^{\prime}$ & $\varphi=\operatorname{average}\left(\arcsin \left(\frac{Y_{0}}{X_{\max }}\right) \mid \arcsin \left(\frac{Y_{0}^{\prime}}{X_{\max }^{\prime}}\right)\right)$ \\
\hline Method $1 X^{\prime}-1 Y^{\prime}$ & $\left.\varphi=\arcsin \left(\frac{X_{0}^{\prime}}{X_{\max }^{\prime}}\right)\right)$ \\
\hline
\end{tabular}

TABLE 2: Phase shift calculated with the different Lissajous based methods in terms of averaged value and standard deviation.

\begin{tabular}{|c|c|c|c|c|c|c|c|c|c|c|}
\hline \multirow{2}{*}{$\mathrm{O}_{2}$ concentration } & \multicolumn{2}{|c|}{$1 X$} & \multicolumn{2}{|c|}{$2 X$} & \multicolumn{2}{|c|}{$1 Y$} & \multicolumn{2}{|c|}{$2 Y^{\prime}$} & \multicolumn{2}{|c|}{$1 X^{\prime}-1 Y^{\prime}$} \\
\hline & $\mu\left({ }^{\circ}\right)$ & $\sigma\left(^{\circ}\right)$ & $\mu\left({ }^{\circ}\right)$ & $\sigma\left(^{\circ}\right)$ & $\mu\left({ }^{\circ}\right)$ & $\sigma\left(^{\circ}\right)$ & $\mu\left({ }^{\circ}\right)$ & $\sigma\left(^{\circ}\right)$ & $\mu\left({ }^{\circ}\right)$ & $\sigma\left(^{\circ}\right)$ \\
\hline $0 \%$ & 9.02 & 0.23 & 9.07 & 0.12 & 9.05 & 0.17 & 9.17 & 0.29 & 9.01 & 0.09 \\
\hline $2 \%$ & 8.44 & 0.21 & 8.60 & 0.21 & 7.97 & 0.23 & 8.33 & 0.31 & 8.23 & 0.11 \\
\hline $7.5 \%$ & 6.68 & 0.18 & 7.33 & 0.14 & 6.82 & 0.24 & 7.24 & 0.26 & 6.87 & 0.11 \\
\hline $15 \%$ & 5.46 & 0.16 & 6.23 & 0.13 & 4.78 & 0.26 & 5.66 & 0.28 & 5.42 & 0.11 \\
\hline $60 \%$ & 3.62 & 0.21 & 4.52 & 0.16 & 3.71 & 0.28 & 4.10 & 0.36 & 3.86 & 0.15 \\
\hline $100 \%$ & 2.63 & 0.34 & 2.60 & 0.31 & 3.56 & 0.52 & 2.07 & 0.59 & 3.09 & 0.13 \\
\hline
\end{tabular}

the shift produced by the quenching effect and, in this manner, calculate the lifetime of the emission. To get this baseline, the excitation signal was allowed to pass through the filter, and a naked fiber optic pigtail was used as sensor. In this manner, it was possible to measure the phase shift induced by the circuit in the excitation signal, which is $67^{\circ}$ (this parameter was obtained with the method that has shown the best performance, which will be indicated later). Thus, this value was subtracted from the measured values, which allowed the real phase shift to be calculated.

In order to evaluate the different methods, Sensor A was exposed to distinct oxygen concentrations, specifically $0 \%, 2 \%, 7.5 \%, 15 \%, 60 \%$, and $100 \%$. The most critical conditions were under a $100 \%$ oxygen concentration because the luminescence signal got highly quenched. The registration and processing of the signals were performed by a Labview virtual instrument: in this manner, the value of the phase shift between signals was determined on real time by the different methods while the working conditions changed. Each concentration was kept for 5 minutes, and the phase shift was calculated every second. The average and standard deviation values while the concentration was constant were used to evaluate the proposed methods. The results obtained are plotted in Figure 4(a) and detailed in Table 2.

In Figure 4(a) it can be observed that the temporal evolution of the phase shift that shows lower fluctuations is the one obtained with the $1 X^{\prime}-1 Y^{\prime}$ method. The methods that use the values of $X_{\max }, X_{\max }^{\prime}$ yield better results in terms of standard deviation than the ones based on $Y_{\max }$ or $Y_{\max }^{\prime}$ : at these points, the deviation in the Lissajous curve is high and the resulting phase shifts show the highest standard deviation values even in the case of $2 Y^{\prime}$ (which uses $X_{\max }, X_{\max }^{\prime}$ ). On the contrary, the approaches based on $X_{\max }$ and $X_{\max }^{\prime}$ offer lower deviations. However, it is important to remark that although methods $1 X$ and $2 X$ offer a good precision, they only take into account the crossing points in the $O X$ axis, so that the accuracy could be poor. In the case of method $1 X^{\prime}-1 Y^{\prime}, X_{\max }$, $X_{\max }^{\prime}$ are used instead of $Y_{\max }$ and $Y_{\max }^{\prime}$, as well as the averaged value of $Y_{0}$ and $Y_{0}^{\prime}$ : on one hand, the error from $X$ crossing points is reduced; on the other hand, averaging a phase shift obtained from $X_{0}$ and $X_{0}^{\prime}$ with another one calculated with $Y_{0}$ and $Y_{0}^{\prime}$ enhances the accuracy of the method. As a result, the lowest error is obtained for this method, as it can be checked in Figure 4(b) and in Table 2: it is significant that, for the $100 \%$ oxygen concentration, the standard deviation is half of the result obtained with $1 X$ method and three times lower compared with $1 Y$ approach.

\section{Experimental Results and Discussion}

To verify the validity of the proposed method, two different experiments were carried out: the first one consists of the calibration of Sensor A and Sensor B following zero crossing approach and $1 X^{\prime}-1 Y^{\prime}$ method (results obtained with FFT were similar to the ones registered with this traditional approach). The second sensor showed a lower emission intensity: to get a similar signal level for both sensors at the spectrometer, integration time had to be set at $750 \mathrm{~ms}$ for Sensor B, whereas for Sensor A it was $25 \mathrm{~ms}$. In the second test, Sensor A was studied under different conditions, 


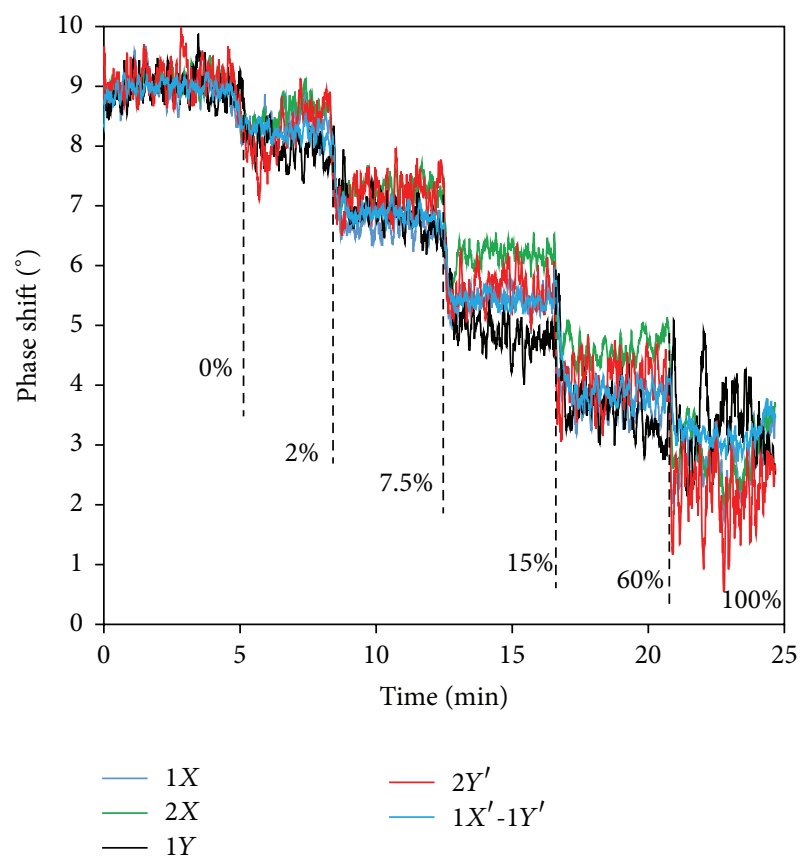

(a)

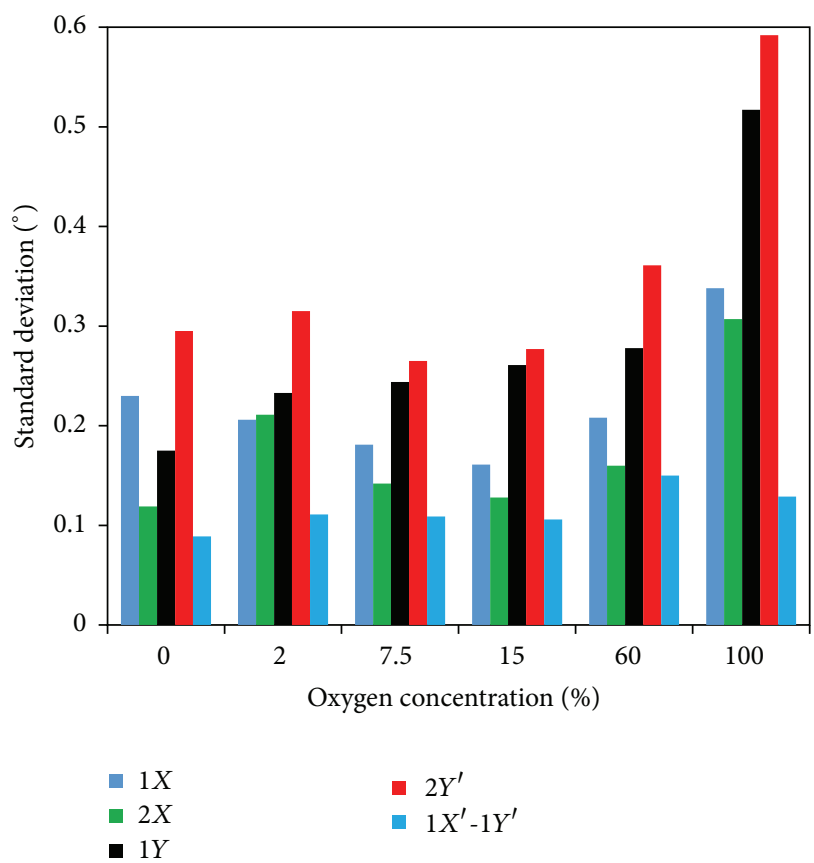

(b)

FIGURE 4: (a) Temporal response from Sensor A when exposed to different oxygen concentrations in terms of the phase shift calculated with the proposed methods based on Lissajous representation. (b) Standard deviation for each method at the different concentrations.

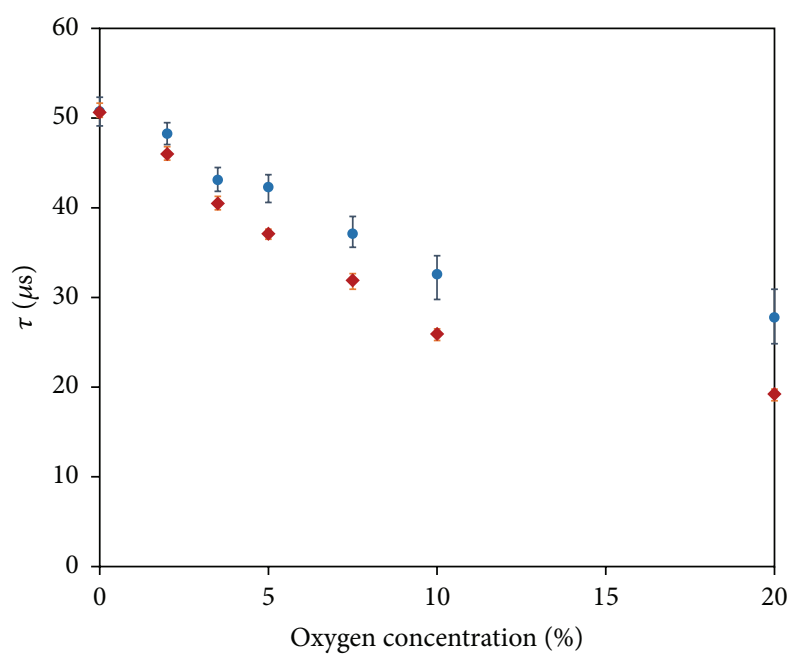

- Zero crossing - $1 X^{\prime}-1 Y^{\prime}$ Lissajous

(a)

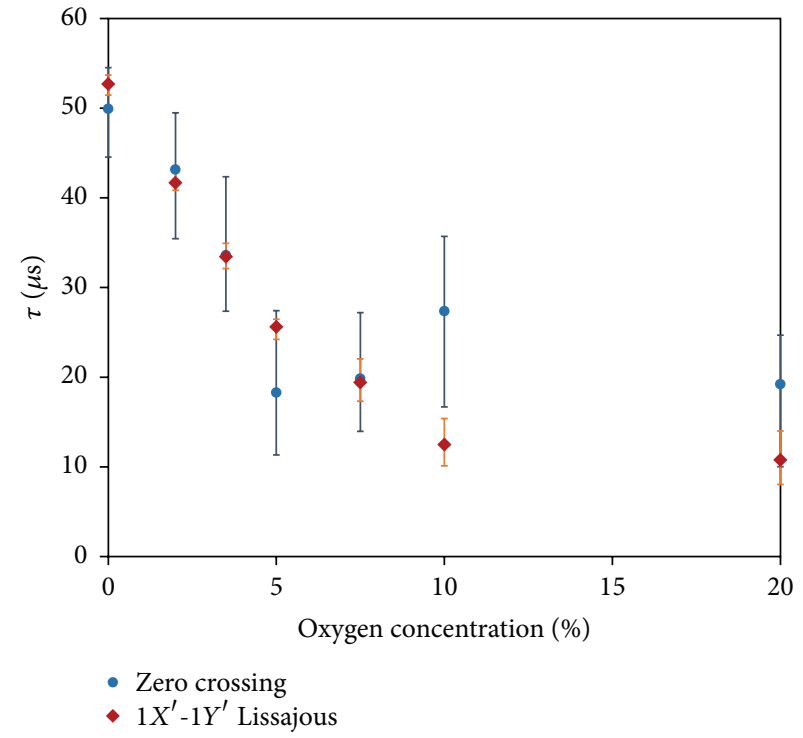

(b)

FIGURE 5: Comparison between the lifetime parameter estimated with zero crossing and $1 X^{\prime}-1 Y^{\prime}$ methods for (a) Sensor A and (b) Sensor B.

specifically reducing the signal level of the excitation light $70 \%$ : in this manner, it was possible to check if the proposed system is independent on the amplitude of the signals.

\subsection{Comparison between Digitalized Lissajous Based Method} and Zero Crossing Estimation. The response of the sensors was analyzed for oxygen concentrations between $0 \%$ and $20 \%$, where the sensitivity was higher for both of them.
The registered data were processed following the zero crossing approach and the $1 X^{\prime}-1 Y^{\prime}$ procedure. For both cases, the calculated phase shift was used to determine lifetime emission applying (1). Figure 5(a) displays the results in terms of lifetime for Sensor A and Figure 5(b) does so for Sensor B. In the case of Sensor A, the results calculated for the different concentrations show a similar trending for both approaches. The deviation obtained from the zero crossing 


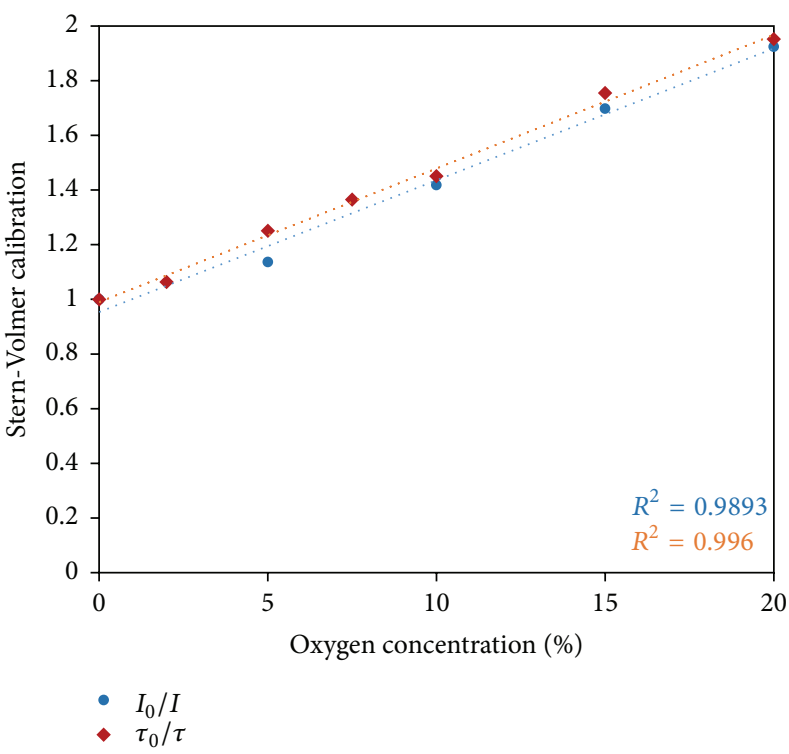

(a)

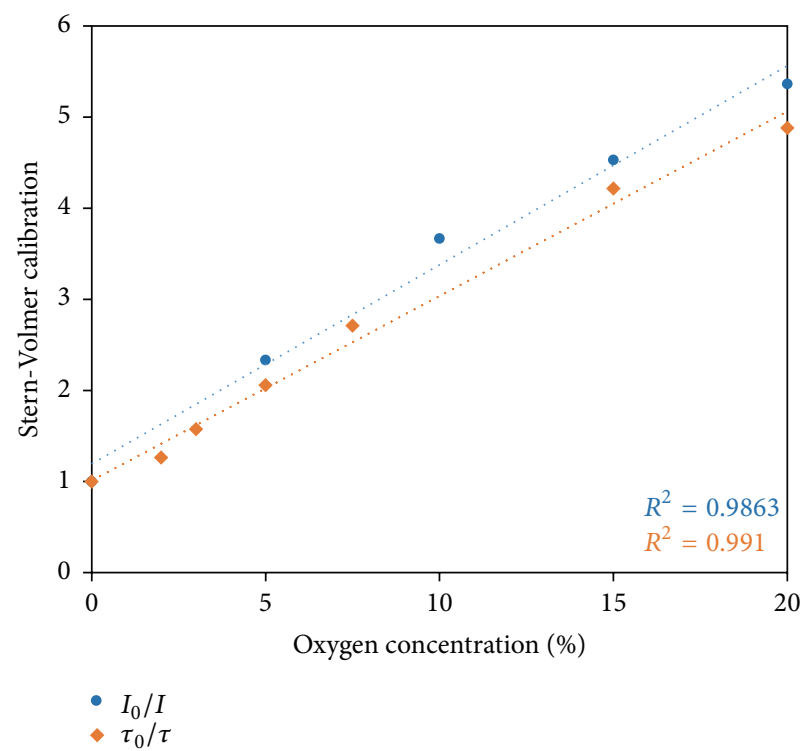

(b)

FIGURE 6: Stern-Volmer representation of the estimated lifetime parameter calculated with method $1 X^{\prime}-1 Y^{\prime}$ compared to the one using intensity values for (a) Sensor A and (b) Sensor B. Inset, the $R^{2}$ factor when applying by LMS.

method increases from $1.5 \mu$ s for $0 \%$ concentration up to $3.0 \mu$ s when $\mathrm{O}_{2}$ is set at $20 \%$; however, the deviation observed when the proposed method was applied is around $0.7 \mu$ s for every case: it performs better in terms of standard deviation because it is, at least, half compared to the best one registered with zero crossing approach. Sensor B scenario was different because the intensity level of the luminescent signal was significantly lower: as it can be observed in Figure 5(b), the phase shift obtained by zero crossing approach does not follow any trend. On the contrary, in the case of the Lissajous method, the error remains always below $3 \mu$ s even as the oxygen concentration increases and, what is more, the obtained values describe a trending curve, so that $1 X^{\prime}$ $1 Y^{\prime}$ method is applicable to sensors with low signal levels. Regarding the standard deviation, in the worst case, applying the zero crossing is up to 8 times higher than the one obtained with the $1 X^{\prime}-1 Y^{\prime}$ method (the detailed standard deviation data are available as Supplementary Material; results from FFT were similar to the ones registered with zero crossing).

In light of these results, it can be inferred that when the signal level is high enough, although both methods offer similar values, the proposed one yields a higher accuracy; moreover, when the amplitude of the sensor signal is low, the zero crossing method is not reliable, whereas $1 X^{\prime}-1 Y^{\prime}$ performs properly. For the probes under study, Sensor B shows a higher dynamic range in terms of lifetime emission, so that it offers a better sensitivity: therefore, method $1 X^{\prime}-1 Y^{\prime}$ allows working with the sensor that although it has a lower signal level shows a better sensitivity.

5.2. Validation of the Lissajous Based Method $1 X^{\prime}-1 Y^{\prime}$. Luminescent sensors can be characterized by means of light intensity and luminescence lifetime: both types of measurements were carried out and compared because if both of them are correct, their calibration curves must be similar and agree with the Stern-Volmer equation:

$$
\frac{I_{0}}{I}=\frac{\tau_{0}}{\tau}=1+K_{\mathrm{SV}}\left[\mathrm{O}_{2}\right] .
$$

In order to validate the proposed method, the sensors under study were calibrated in terms of (7) using intensity and lifetime emission data: as it can be seen in the equation, the calibration obtained with both types of measurements (based on intensity and lifetime, resp.) should yield similar $K_{\mathrm{SV}}$ constant. Therefore, intensity based characterization was performed just to validate the lifetime measurements. To achieve it, the high pass filter, the photomultiplier, and the A/D converter were substituted by a spectrometer (USB2000FLG from Ocean Optics Inc.).

The calibration lines obtained for each device following Stern-Volmer equation are plotted in Figure 6(a) (Sensor A) and in Figure 6(b) (Sensor B). It can be observed that although there is a compensable offset, the sensitivity is similar when calibrating the sensors with intensity or lifetime information. Therefore, the Lissajous based method allows us to calibrate the sensors in a reliable way. Moreover, as it was reported in the previous section, Sensor B has higher sensitivity, which matches the lifetime dynamic range observed in Figure 5(b). The trending lines obtained by Least Mean Square (LMS) method are detailed in Table 3.

As the signal from Sensor A was higher, it was decided to calibrate it attenuating the LED source in order to check if the intensity level of the signal has no effect on the lifetime measurements. To verify this point, a filter which induced $70 \%$ intensity losses was placed after the light source. The sensor was exposed again to different oxygen concentrations as in previous experiments. Regarding the calculated lifetime emission, the results matched the ones obtained with 
TABLE 3: Comparison between the calibration expressions for both sensors using intensity and lifetime information.

\begin{tabular}{llr}
\hline & \multicolumn{1}{c}{ Sensor A } & Sensor B \\
\hline$\frac{I_{0}}{I}=1+K_{\mathrm{SV}}\left[\mathrm{O}_{2}\right]$ & $\frac{I_{0}}{I}=0.953+0.048\left[\mathrm{O}_{2}\right]$ & $\frac{I_{0}}{I}=1.192+0.215\left[\mathrm{O}_{2}\right]$ \\
\hline$\frac{\tau_{0}}{\tau}=1+K_{\mathrm{SV}}\left[\mathrm{O}_{2}\right]$ & $\frac{\tau_{0}}{\tau}=0.960+0.049\left[\mathrm{O}_{2}\right]$ & $\frac{\tau_{0}}{\tau}=1.001+0.204\left[\mathrm{O}_{2}\right]$ \\
\hline
\end{tabular}

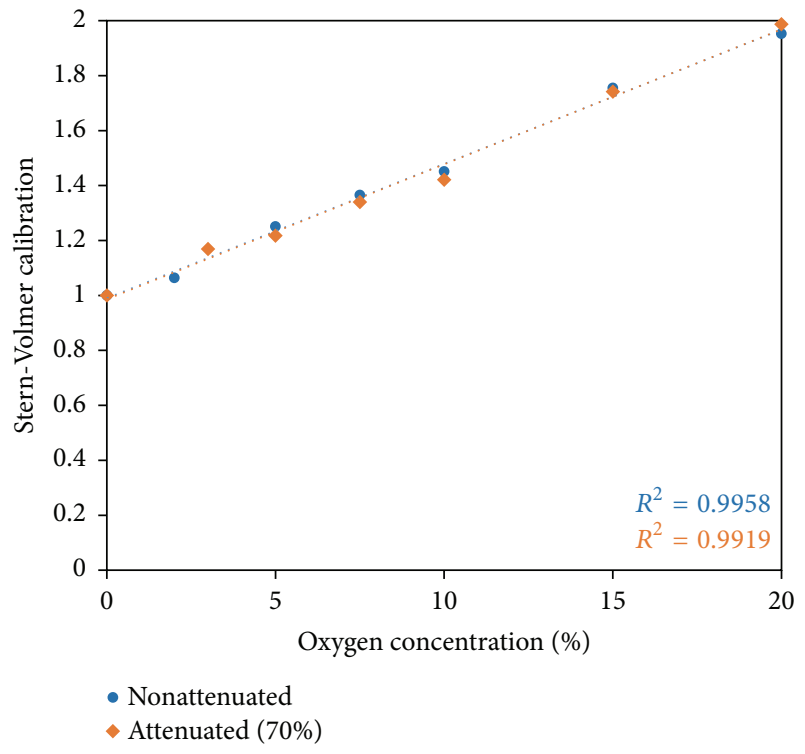

FIGURE 7: Stern-Volmer calibrations obtained for the same sensor working at different signal levels.

the higher signal level: it can be checked in Figure 7. Therefore, the proposed method allows the sensor to be calibrated with no dependence on signal level, which validates its operation.

\section{Conclusions}

An improved method has been proposed to characterize luminescence based sensors in terms of the phase shift between the exciting signal and the luminescent emission from the sensor, and this parameter was used to estimate the emission lifetime. Phase shift parameter is obtained by digital signal processing of both signals at the points that show a lower noise deviation: this information is processed in the digital domain to get the best accuracy and precision. In this manner, the modulation, acquisition, and processing of the different signals could be embedded in a single system to enhance the features of the proposed approach.

The Lissajous parametrical representation in the digital domain is employed to estimate the phase shift, using the points that show the smallest deviation. Compared with traditional approaches, the proposed procedure offers better results in terms of accuracy and precision. What is more important, in the case of the sensors with low signal level, $1 X^{\prime}-1 Y^{\prime}$ method allows handling with low intensity signal sensors and calibrating them properly. The lifetime calibrations obtained match the ones based on intensity for the studied sensors. Moreover, one of them was studied with different signal levels, and it is observed that the calibration is not affected by this intensity change. These tests validate the performance of the method.

\section{Competing Interests}

The authors declare that they have no competing interests.

\section{Acknowledgments}

This work was supported in part by the Spanish Ministry of Economy and Competitiveness FEDER TEC2013-43679-R.

\section{References}

[1] M. Bourmpos, A. Argyris, and D. Syvridis, "Smart city surveillance through low-cost fiber sensors in metropolitan optical networks," Fiber and Integrated Optics, vol. 33, no. 3, pp. 205223, 2014.

[2] A. D. Kersey, "A review of recent developments in fiber optic sensor technology," Optical Fiber Technology, vol. 2, no. 3, pp. 291-317, 1996.

[3] M. Pospíšilová, G. Kuncová, and J. Trögl, "Fiber-optic chemical sensors and fiber-optic bio-sensors," Sensors, vol. 15, no. 10, pp. 25208-25259, 2015.

[4] X. Wang and O. S. Wolfbeis, "Optical methods for sensing and imaging oxygen: materials, spectroscopies and applications," Chemical Society Reviews, vol. 43, no. 10, pp. 3666-3761, 2014.

[5] M. Schäferling, "The art of fluorescence imaging with chemical sensors," Angewandte Chemie-International Edition, vol. 51, no. 15, pp. 3532-3554, 2012.

[6] S. B. Bambot, G. Rao, M. Romauld et al., "Sensing oxygen through skin using a red diode laser and fluorescence lifetimes," Biosensors and Bioelectronics, vol. 10, no. 6-7, pp. 643-652, 1995.

[7] C. Elosua, N. de Acha, D. Lopez-Torres, I. R. Matias, and F. J. Arregui, "Luminescent optical fiber oxygen sensor following layer-by-layer method," Procedia Engineering, vol. 87, pp. 987990, 2014.

[8] O. S. Wolfbeis, Fiber Optic Chemical Sensors and Biosensors, CRC Press, Boca Raton, Fla, USA, 1991.

[9] M. Valledor, J. C. Campo, I. Sánchez-Barragán, J. C. Viera, J. M. Costa-Fernández, and A. Sanz-Medel, "Luminescent ratiometric method in the frequency domain with dual phase-shift measurements: application to oxygen sensing," Sensors and Actuators, B: Chemical, vol. 117, no. 1, pp. 266-273, 2006.

[10] M. A. Bopp, Y. Jia, L. Li, R. J. Cogdell, and R. M. Hochstrasser, "Fluorescence and photobleaching dynamics of single lightharvesting complexes," Proceedings of the National Academy of Sciences of the United States of America, vol. 94, no. 20, pp. 10630-10635, 1997. 
[11] M. E. Lippitsch, S. Draxler, and D. Kieslinger, "Luminescence lifetime-based sensing: new materials, new devices," Sensors and Actuators B: Chemical, vol. 38, no. 1-3, pp. 96-102, 1997.

[12] Y. Kostov, P. Harms, and G. Rao, "Ratiometric sensing using dual-frequency lifetime discrimination," Analytical Biochemistry, vol. 297, no. 1, pp. 105-108, 2001.

[13] B. B. Collier and M. J. McShane, "Time-resolved measurements of luminescence," Journal of Luminescence, vol. 144, pp. 180-190, 2013.

[14] P. A. S. Jorge, P. Caldas, C. C. Rosa, A. G. Oliva, and J. L. Santos, "Optical fiber probes for fluorescence based oxygen sensing," Sensors and Actuators, B: Chemical, vol. 103, no. 1-2, pp. 290299, 2004.

[15] K. T. V. Grattan and Z. Zhang, Fiber Optic Fluorescence Thermometry, Chapman \& Hall, London, UK, 1995.

[16] Y. Yan, C. Petchprayoon, S. Mao, and G. Marriott, "Reversible optical control of cyanine fluorescence in fixed and living cells: optical lock-in detection immunofluorescence imaging microscopy," Philosophical transactions of the Royal Society of London-Series B: Biological sciences, vol. 368, no. 1611, Article ID 20120031, 2013.

[17] S. Medina-Rodríguez, Á. de la Torre-Vega, C. Medina-Rodríguez, J. F. Fernández-Sánchez, and A. Fernández-Gutiérrez, "On the calibration of chemical sensors based on photoluminescence: selecting the appropriate optimization criterion," Sensors and Actuators B: Chemical, vol. 212, pp. 278-286, 2015.

[18] F. Baldini, A. N. Chester, J. Homola, and S. Martellucci, Optical Chemical Sensors, vol. 224 of NATO Science Series, 2003, http://www.scopus.com/inward/record.url?eid=2-s2.040449135046\&partnerID=tZOtx3yl.

[19] C.-S. Chu and Y.-L. Lo, "Ratiometric fiber-optic oxygen sensors based on sol-gel matrix doped with metalloporphyrin and 7amino-4-trifluoromethyl coumarin," Sensors and Actuators, B: Chemical, vol. 134, no. 2, pp. 711-717, 2008.

[20] P. J. Cywinski, A. J. Moro, S. E. Stanca, C. Biskup, and G. J. Mohr, "Ratiometric porphyrin-based layers and nanoparticles for measuring oxygen in biosamples," Sensors and Actuators, $B$ : Chemical, vol. 135, no. 2, pp. 472-477, 2009.

[21] C.-S. Chu and S.-W. Chu, "Portable optical oxygen sensor based on time-resolved fluorescence," Applied Optics, vol. 53, no. 32, pp. 7657-7663, 2014.

[22] C.-S. Chu and Y.-L. Lo, "Highly sensitive and linear calibration optical fiber oxygen sensor based on Pt(II) complex embedded in sol-gel matrix," Sensors and Actuators, B: Chemical, vol. 155, no. 1, pp. 53-57, 2011.

[23] C.-S. Chu, Y.-L. Lo, and T.-W. Sung, "Review on recent developments of fluorescent oxygen and carbon dioxide optical fiber sensors," Photonic Sensors, vol. 1, no. 3, pp. 234-250, 2011.

[24] C. Elosua, C. Bariain, I. R. Matias et al., "Pyridine vapors detection by an optical fibre sensor," Sensors, vol. 8, no. 2, pp. 847-859, 2008.

[25] F. J. Sainz-Gonzalo, C. Popovici, M. Casimiro et al., "A novel tridentate bis(phosphinic acid)phosphine oxide based europium(iii)-selective Nafion membrane luminescent sensor," Analyst, vol. 138, no. 20, pp. 6134-6143, 2013.

[26] G. Decher, "Fuzzy nanoassemblies: toward layered polymeric multicomposites," Science, vol. 277, no. 5330, pp. 1232-1237, 1997.

[27] F. Hua and Y. M. Lvov, "Layer-by-layer assembly," in The New Frontiers of Organic and Composite Nanotechnology, chapter 1, pp. 1-44, Elsevier, 2008.
[28] C. Elosua, N. De Acha, M. Hernaez, I. R. Matias, and F. J. Arregui, "Layer-by-Layer assembly of a water-insoluble platinum complex for optical fiber oxygen sensors," Sensors and Actuators, B: Chemical, vol. 207, pp. 683-689, 2015.

[29] V. I. Arnold, Mathematical Methods of Classical Mechanics, vol. 60 of Graduate Texts in Mathematics, 2003, http://www .scopus.com/inward/record.url?eid=2-s2.0-84861591712\&partnerID=tZOtx3y1.

[30] M. L. Sanderson, "Electrical measurements," in Instrumentation Reference Book, W. Boyes, Ed., chapter 27, pp. 439-498, Elsevier, San Diego, Calif, USA, 4th edition, 2010. 


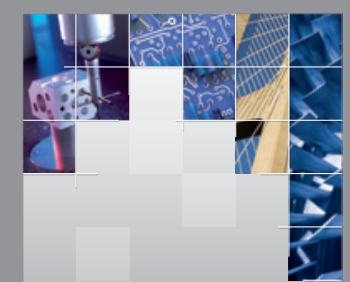

\section{Enfincering}
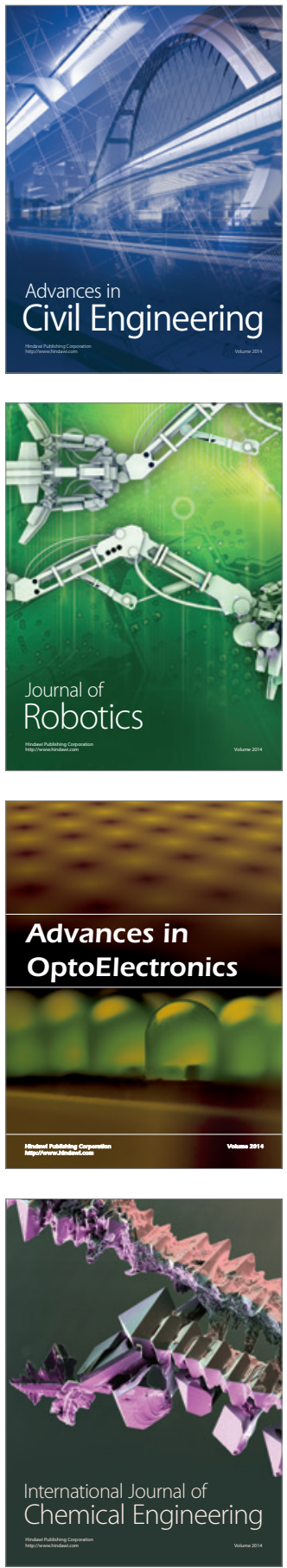

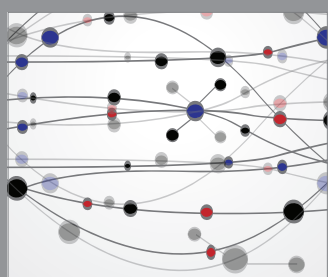

The Scientific World Journal

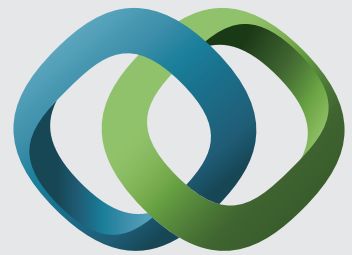

\section{Hindawi}

Submit your manuscripts at

http://www.hindawi.com
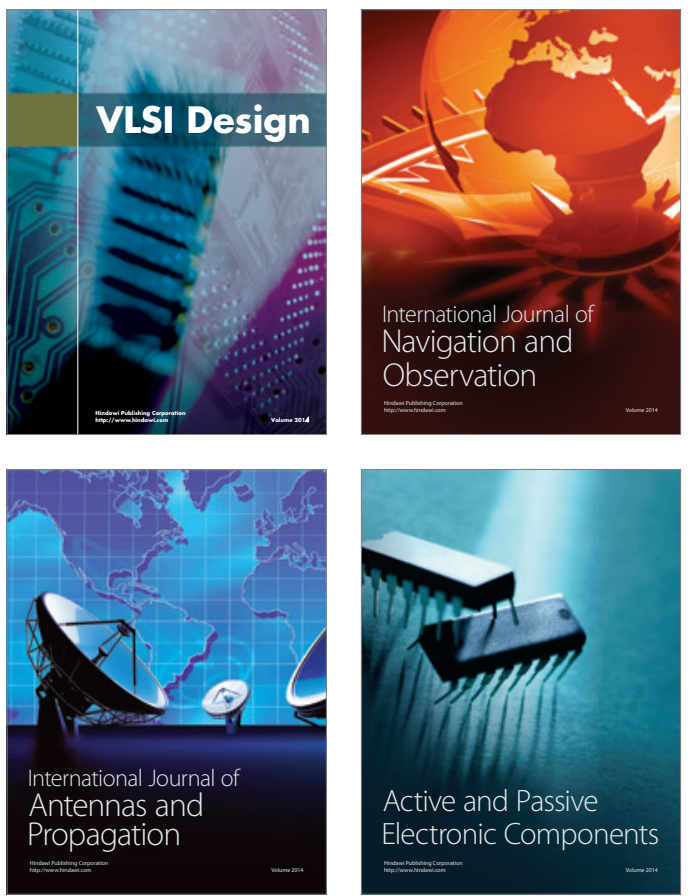
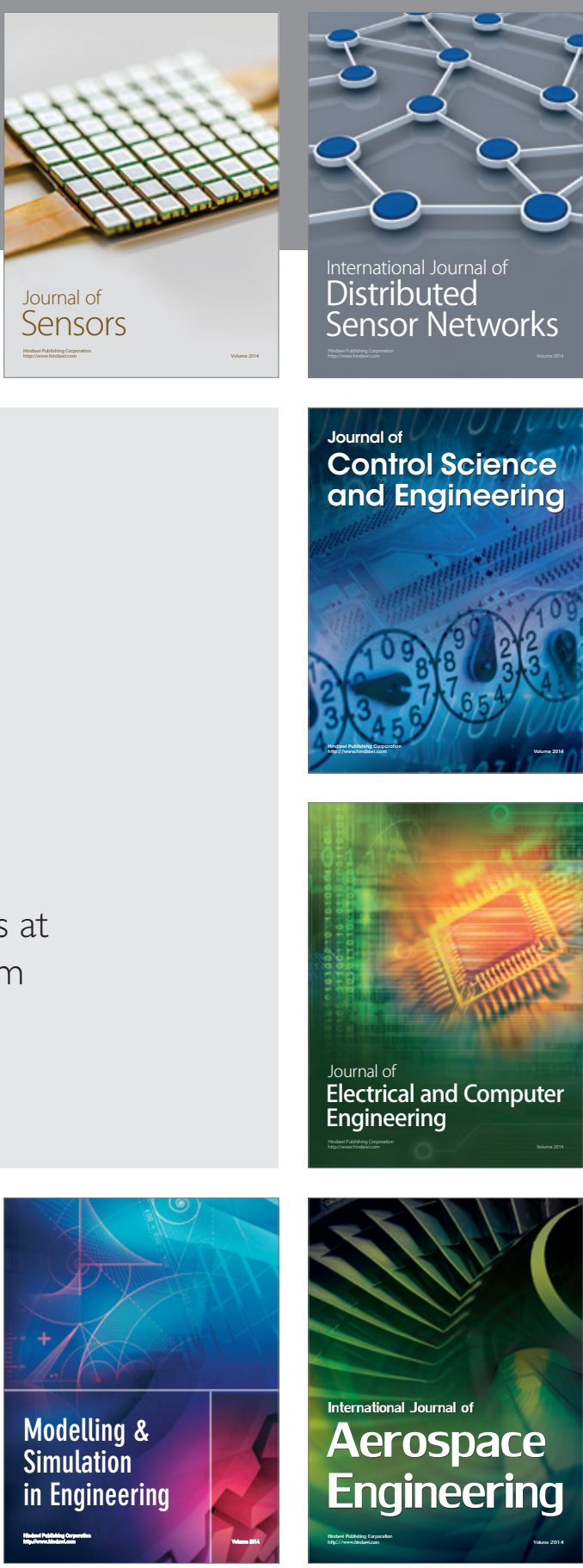

International Journal of

Distributed

Sensor Networks

Journal of

Control Science

and Engineering
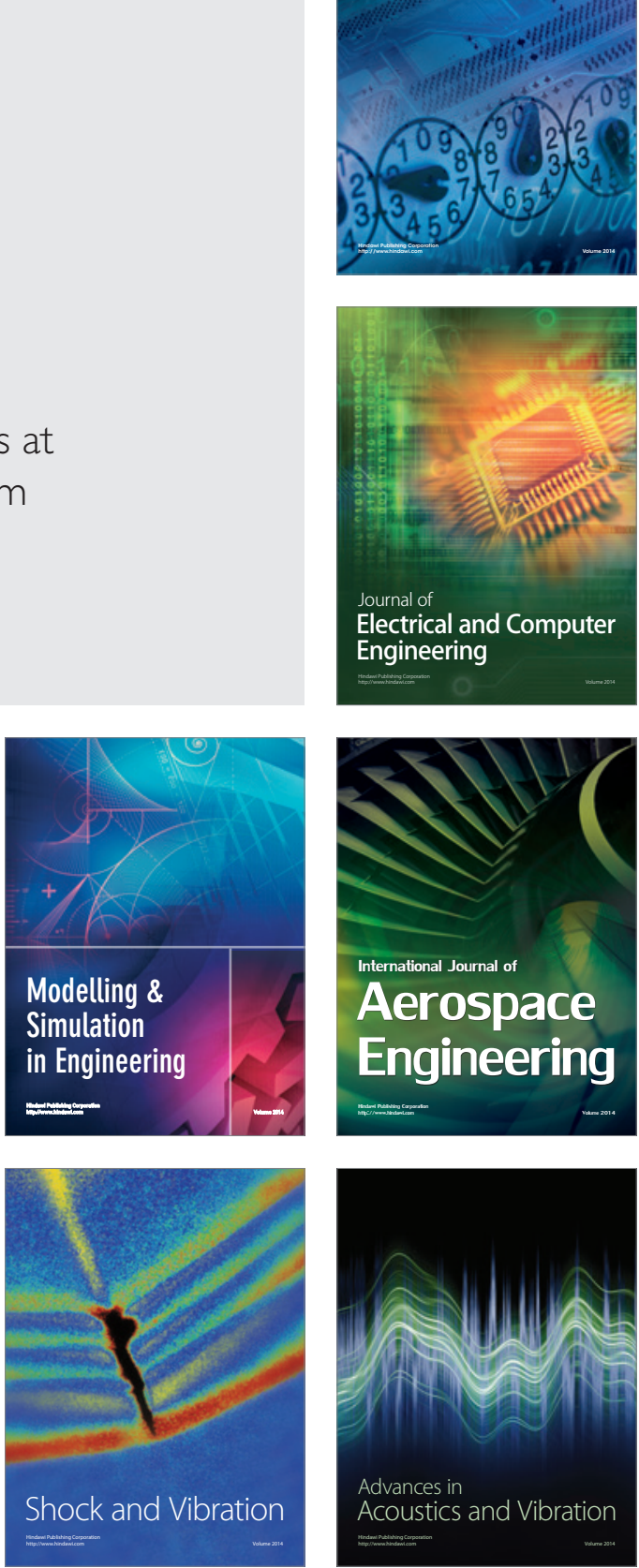